\title{
Investigating the ability of various building in handling load shiftings
}

\author{
Paul Malisani* ${ }^{\ddagger}$, Bérenger Favre ${ }^{\dagger}$, Stéphane Thiers ${ }^{\dagger}$, Bruno Peuportier ${ }^{\dagger}$, François Chaplais ${ }^{\ddagger}$, Nicolas Petit $\ddagger$ \\ ${ }^{*}$ EDF R\&D \\ ${ }^{\dagger}$ CEP - MINES-ParisTech \\ ${ }^{\ddagger} \mathrm{CAS}$ - MINES-ParisTech \\ Emails: firstname.name@mines-paristech.fr
}

\begin{abstract}
In modern constructions, several insulation configurations and technologies exist for residential buildings. Therefore, during renovation of these buildings, various investments can be considered. In this article, we consider building equipped with electric heaters. The contribution of this article is a method for evaluating the ability of each configuration to keep the inhabitants comfortable during load shifting periods. This question is of importance in the relationship, and then in the price setting, between the user (inhabitant of the house) and the energy provider who uses these load shifting periods to optimize his production on a regional or national scale.

We proceed as follows: an optimization method is used to compute, in a dynamical context, the best heating strategy. The weather conditions and the comfort constraints define (through the solution of the dynamical optimization problem) the actual ability of the building to guarantee a satisfying comfort during load shifting periods independently on the regulation strategy.

The conclusion is that for poorly insulated building $(\approx 58 \%$ of the french stock) it is impossible to achieve load shifting superior to 20 minutes in winter time, even when using advanced regulation strategy of the heating system.
\end{abstract}

\section{INTRODUCTION}

Recently significant efforts have been made to reduce electricity peak demand. In Europe these peaks mostly occur during winter time, and are mainly due to heating systems. In order to guarantee the grid stability, numerous studies are carried out on the overall load reduction. At the level of the individual houses, this reduction can be achieved thanks to a careful building design that can efficiently capture and restore the solar gains [1]. An advanced heating control strategy also be a solution. Such control can be based on power tariff [2] and can use the building thermal mass as an asset to shift the building consumption, reducing the peak consumption [3], [4] in turn.

This article follows this approach and studies the load shifting opportunities on five thermal models ranging from poorly to well insulated buildings. The contribution consists in a dynamic optimization under constraints method in continuous time allowing to accurately compute optimal trajectories (when they exist) [5], [6], [7], [8]. By acting on the duration of load shifting, one can figure out the maximum duration of a complete heating load shifting while maintaining an acceptable level of comfort and shifting the consumption. The results obtained in this study show that the thermal mass of a poorly insulated building is not sufficient to perform load shiftings superior to ten minutes. Thus, the use of buildings as power stocks only seems relevant in the case of sufficiently insulated buildings which can handle load shiftings of several hours. Practical cases of interest are presented.

In Section II, a description of the considered building is given, together with the numerical method to obtain a high order linear model of the system. In Section III, the reduction method is described together with the constraints of the optimization. In Section IV, the algorithm of constrained dynamical optimization is presented. In Section $\mathrm{V}$, the results on the abilities of the different considered system are presented together with the maximum bearable duration of daily load shiftings for each model. Finally in Section VI the conclusion and the perspectives of the study are presented.

\section{BUILding MODEL}

\section{A. Building description}

The building under study is a single-family house. It corresponds to an actual experimental passive house being part of the INCAS platform built in Le Bourget du Lac, France (see Figure 1). For our study, five low performance versions of the building are considered. The reference version corresponds to a house built before the first French thermal regulation (1975). This reference version used to represent $58 \%$ of the french stock in 2008. The four other versions correspond to different renovation levels of the reference. It is then possible to study the effects of renovation on the peak load management. The reference has two floors for a total living area of $89 \mathrm{~m}^{2}$. Only $34 \%$ of its south facade surface is glazed while the north facade has only two small windows. All the windows are single-glazed. The south facade is also equipped with solar protections for the summer period. The external walls are made of a $30 \mathrm{~cm}$-thick layer of concrete blocks and the floor is composed of $20 \mathrm{~cm}$ reinforced concrete. There is no insulation in the building except for the $10 \mathrm{~cm}$ of glass-wool in the attic. According to thermal simulation results using the software Pléiades+COMFIE [9], the heating load is $253 \mathrm{kWh} /\left(\mathrm{m}^{2}\right.$.year) which is typical for such type of house. Comparisons have been performed during the design phase on the passive house version of this building with other simulation tools like Energy Plus and TRNSYS [10] and have shown similar results.

Four different renovations of this building are presented in Table I: 


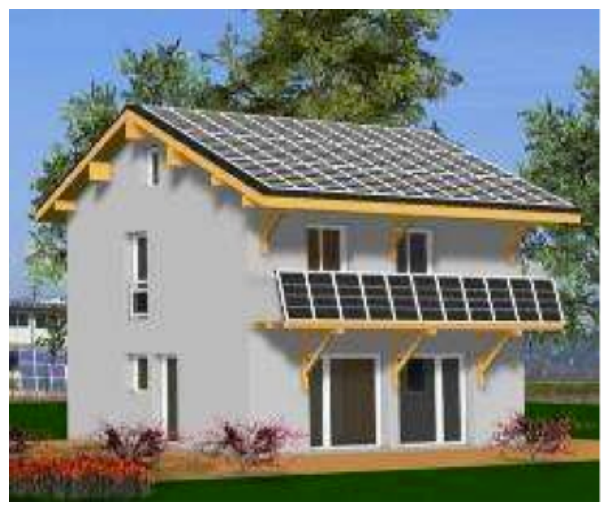

Fig. 1. 3D view of the house (west and south facades)

TABLE I

VERSIONS OF THE CONSIDERED BUILDINGS THROUGHOUT THE RENOVATIONS WORKS

\begin{tabular}{|c|c|c|}
\hline Version & $\begin{array}{l}\text { Renovation } \\
\text { applied }\end{array}$ & $\begin{array}{c}\text { Heating } \\
\text { consumption } \\
\left(\mathrm{kWh} / \mathrm{m}^{2} \text {.year }\right)\end{array}$ \\
\hline $\begin{array}{l}\text { Reference } \\
\left(1^{\mathrm{st}}\right)\end{array}$ & none & 253 \\
\hline $\begin{array}{c}\text { Roof } \\
\text { insulation } \\
\left(2^{\text {nd }}\right)\end{array}$ & $\begin{array}{c}(1)+30 \mathrm{~cm} \text { of } \\
\text { glass-wool in the attic }\end{array}$ & 246 \\
\hline $\begin{array}{c}\text { Triple glazing } \\
\left(3^{\text {rd }}\right)\end{array}$ & $\begin{array}{c}(2)+\text { Triple glazed } \\
\text { windows }\end{array}$ & 215 \\
\hline $\begin{array}{l}\text { Insulation of } \\
\text { external walls } \\
\left(4^{\text {th }}\right)\end{array}$ & $\begin{array}{l}(3)+15 \mathrm{~cm} \text { of glass- } \\
\text { wool in external walls }\end{array}$ & 93 \\
\hline $\begin{array}{c}\text { Heat recovery } \\
\text { ventilation (HRV) } \\
\left(5^{\text {th }}\right)\end{array}$ & $\begin{array}{c}\text { (4) + HRV with an } \\
\text { efficiency of } 0.5 \\
\text { (accounting for air infiltration) }\end{array}$ & 80 \\
\hline
\end{tabular}

\section{B. Thermal model}

The building is modeled as spatial zones of homogenous temperature. For each zone, each walls are divided in fine meshes small enough to also have a homogeneous temperature. There is one more mesh for the air and furniture in the zone. Eventually, a thermal balance is done on each mesh within the building. It takes into account:

- $P_{\text {cond }}$ the losses (or gains) by conduction in walls, floor and ceiling.

- $P_{\text {sol }}$ : the gains due to solar irradiance through the windows

- $P_{\text {conv }}$ : the losses (or gains) due to convection at walls surface

- $P_{\text {in }}$ : the internal gains due to heating, occupancy and other loads (only for zone air mesh)

- $P_{\text {bridges }}$ : heat losses through thermal bridges, not associated to thermal mass

- $P_{\text {ventil }}$ : heat losses due to air exchange

When applied to the mesh corresponding to the air of each zone, the thermal balance equation is:

$$
C_{\text {air }} \dot{T}_{\text {air }}=P_{\text {in }}+P_{\text {cond }}+P_{\text {bridges }}+P_{\text {ventil }}+P_{\text {sol }}+P_{\text {conv }}
$$

with the thermal capacity of the node air (including furniture) and the temperature of the mesh. For each zone, repeating equation (1) for each mesh and adding an output equation leads to the following continuous linear time-invariant system.

$$
\left.\begin{array}{l}
C \dot{T}(t)=A T(t)+E U(t) \\
Y(t)=J T(t)+G U(t)
\end{array}\right\}
$$

with:

- $T$ mesh temperatures vector

- $U$ driving forces vector (climate parameters, heating, etc.)

- $Y$ outputs vector (here, temperature of the air nodes)

- $C$ thermal capacity diagonal matrix

- $A, E, J, G$ matrices relating the vectors of the dynamics.

In order to simulate such a model, it is important to know the occupancy of the building, which defines partly $P_{\text {in }}$ with the emission of heat by the inhabitants and the appliances. The second part of heat emission in $P_{\text {in }}$ is due to the heating system. Another important aspect is the weather model. It defines the loss due to heat transfer with the ambient temperature and the gain with solar irradiance. All the data of the occupancy and weather models are contained in the input vector $U$.

\section{MOdEL REDUCTION AND CONSTRAINTS}

\section{A. Model reduction}

A high order linear model (2) is now available. In view of application of optimization methods, its state dimension is too large to allow a fast convergence of the optimization algorithm. A reduction method is applied to lower the state dimension and thus to make the algorithm faster. To reduce the dimension of the dynamics, several methods exist such as, e.g., singular perturbations [11], or identification methods [12]. In our case, an efficient method is the balanced truncation [13]. Indeed, this truncation consists in removing the state variables which receive the effort from the input and contribute the less to the variations of the output, that is to say, the state variables easily negligible from an energetic view-point ${ }^{1}$. To determine the order of the reduced model, one can compare the error between the high order model and the reduced ones over one year in terms of mean and standard deviation. Here, we decide to take the minimal order such that these statistical properties are both inferior to 0.1 . In our case, all models are at least third order, and one of them is fourth order.

In table II, one can find the various time constants of the considered models. It is noticeable that the main effects of the renovation is to enlarge the slow time constant. This is particularly true for the adjunction of insulation. Moreover, one can also see that a thermal building model clearly has three well separated time scales [11].

\section{B. Model and constraints}

1) Model notations: In the following, we use the classical linear state space representation to represent the model:

$$
\begin{aligned}
\dot{x}(t) & =A x(t)+B u(t)+d(t) \\
y(t) & =C x(t)
\end{aligned}
$$

\footnotetext{
${ }^{1}$ We refer the interested reader to [13]
} 
TABLE II

VALUE OF THE TIME CONSTANTS OF THE FIVE DIFFERENT MODELS.

\begin{tabular}{c|c|c|c|c|c|} 
& $1^{\text {st }}$ & $2^{\text {nd }}$ & $3^{\text {rd }}$ & $4^{\text {th }}$ & $5^{\text {th }}$ \\
\hline Time & $8 \mathrm{~min}$. & $7 \mathrm{~min}$. & $8 \mathrm{~min}$. & $9 \mathrm{~min}$. & $9 \mathrm{~min}$. \\
constants & $13 \mathrm{~h}$. & $13 \mathrm{~h}$. & $2 \mathrm{~h}$. & $13 \mathrm{~h}$. & $18 \mathrm{~h}$. \\
& $95 \mathrm{~h}$. & $98 \mathrm{~h}$. & $8 \mathrm{~h}$. & $160 \mathrm{~h}$. & $180 \mathrm{~h}$. \\
& & & $91 \mathrm{~h}$. & &
\end{tabular}

where $x$ is the state of the model, $y$ is the inside temperature, $d$ represents the influence of the outside temperature and the solar fluxes on the heating of the house and $u$ represents the heating flux on the air node and is the control variable.

2) Constraints:

a) Inside temperature constraints: The temperature constraints are 24 hours periodic and are:

- $y \leq 24^{\circ} \mathrm{C}$ at all times.

- $y \geq 14^{\circ} \mathrm{C}$ between 9 a.m. and 5 p.m.

- $y \geq 20^{\circ} \mathrm{C}$ otherwise.

To simplify the notations, we write the temperature constraints above as follows:

$$
y^{-} \leq y \leq y^{+}
$$

b) Control constraints: The control constraints are not the same for all systems:

- $0 \leq u \leq 20 \mathrm{~kW}$ for the buildings whose walls have not been insulated.

- $0 \leq u \leq 10 \mathrm{~kW}$ for the buildings whose walls have been insulated.

To simplify the notations for the algorithm, we write the control constraints as follows:

$$
0 \leq u \leq u^{+}
$$

c) Load shifting: In our case the load shiftings consist in daily time period when the heating of the house is not allowed to consume any energy. These shiftings start everyday at 5 p.m.. The objective of this study is to determine the maximum duration of these load shiftings such that it becomes impossible to satisfy both (5) and (6).

\section{METHOD AND ALGORITHM}

\section{A. Method}

In order to characterize the duration of load shifting which allows the inside temperature to satisfy (5) while the heating power satisfies (6), a state constrained optimal control approach is used. Indeed, setting (5) and (6) as constraints allows us to quantify the maximum load shifting duration feasible without degradation of the comfort. Indeed, when there exists no solution for the constrained optimal control problem, we know that the load shiftings are too long. This is independent of the regulation system but rely on the capacity of the building to store energy.

To determine the maximum duration of the load shifting, a sequence of optimal control problems is solved with increasing load shifting periods until there exists no solution satisfying the constraints (5) and (6).

\section{B. Algorithm}

For this problem, the constraints are (5) and (6) and the considered criterion is the energy consumed over the whole week. To solve the state constraint optimal control problem a maximum principle-based interior method is used [5], [6], [7], [8], [14], which has been adapted for the energy consumption problem. The criterion is given by the following:

$$
J=\min _{u \in\left[0, u^{+}\right]} \int_{0}^{T} u(t) d t
$$

with the dynamics and the state constraint $y \in\left[y^{-}, y^{+}\right]$seen above, and where $T=7$ days.

The first step, in this algorithm is to operate the following change in variable ${ }^{2}$ on the control variable $u$ :

$$
u \triangleq \phi(\nu)=u^{+}\left(\frac{e^{k \nu}}{1+e^{k \nu}}\right)
$$

This change in variable is such that $\nu$ is an unconstrained variable and the optimization problem is now:

$$
J=\min _{\nu \in \mathbb{R}} \int_{0}^{T} \phi(\nu) d t
$$

To solve this problem, an indirect method [16] using an adjoint vector $p$ is used. This adjoint vector $p$ has the same dimension as the state $x$ of the reduced dynamical system (3). It satisfies

$$
\frac{d p}{d t}(t)=-A^{t} p(t)-C^{t} \gamma_{y}^{\prime}(C x(t))
$$

where $\gamma_{y}^{\prime}$ is the derivative of the following function

$$
\gamma_{y}(y(t))=\left(\frac{y^{+}(t)-y^{-}(t)}{\sqrt{\left(y^{+}(t)-y(t)\right)\left(y(t)-y^{-}(t)\right)}}-1\right)^{2.1}
$$

In (11), the expression serves to keep the output away from the constraint (see [15]). The power 2.1 guarantees the wellbehavness of the method (see [8]). Now, to compute the optimal control, an interior method is used. It consists in solving a sequence of optimal control problems, depending on a parameter $\epsilon_{n}$, converging to the optimal solution of the problem. The iterative algorithm is the following:

- Step 1: Initialize the functions $x(t)$ and $p(t)$ such that the initial $C x(t) \in\left(y^{-}(t), y^{+}(t)\right)$ for all $t \in[0, T]$, and set $\epsilon=\epsilon_{0}$. Note that $x(t)$ and $p(t)$ do not need to satisfy any differential equations at this stage, even if it is better if they do. Often, $p$ can be chosen identically equal to zero at first step.

- Step 2: Compute $\nu_{\epsilon}^{*}=\sinh ^{-1}\left(-\frac{1+p^{t} B}{\epsilon}\right)$. Thus the optimal solution $u_{\epsilon}^{*}=\phi^{-1}\left(\nu_{\epsilon}^{*}\right)$ is given using equation (8) with $k=0.8$.

- Step 3: Solve the 2 differential systems of equation $\frac{d x}{d t}=$ $A x+B u_{\epsilon}^{*}$ and $\frac{d p}{d t}=-A^{t} p-C^{t} \gamma_{y}^{\prime}(C x)$ forming a two point boundary value problem using bvp4c (see [17]), with the following boundary constraints $x(0)=x_{0}$ and $p(T)=0$.

\footnotetext{
${ }^{2}$ Similar to the saturation function approach considered in [15]
} 
- Step 4: Decrease $\epsilon$, initialize $x(t)$ and $p(t)$ with the solutions found at Step 3 and restart at Step 2.

In our case, the sequence $\left(\epsilon_{n}\right)$ has been chosen such that $\epsilon_{n}=10^{-\frac{n}{10}}$ with $n=0 \cdots 40$. The proof of convergence of this algorithm can be found in [8]. Details on its derivation can also be found in [15].

\section{Results}

The considered optimization takes place in winter over one particularly cold week where the ambient temperature is given on Figure 2. In Figures 4 and 5, one can see the influence of the load shiftings on the indoor temperature of the buildings.

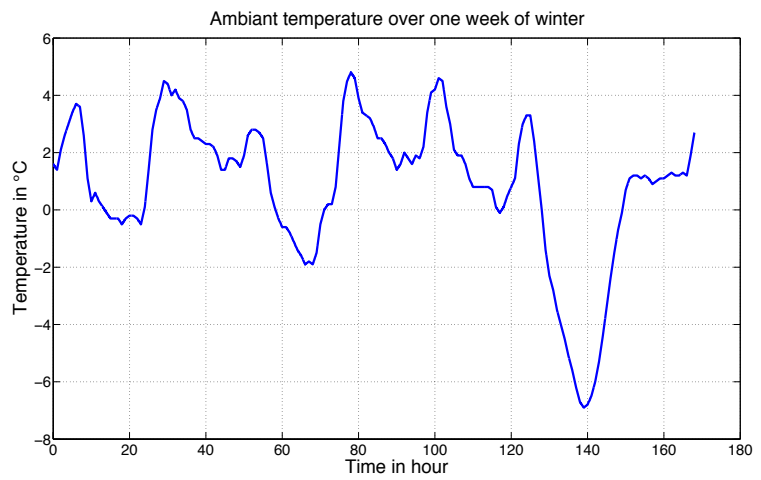

Fig. 2. Ambient temperature over one week of winter

\section{A. Summary of the results}

Figure 3 displays the optimal consumption of energy with the maximum load shifting duration and without load shifting for each building version. In Table III the maximum load shifting duration is exhibited for each version. The behavior of the five system towards the maximum bearable load shifting are displayed in Figures 4 and 5.

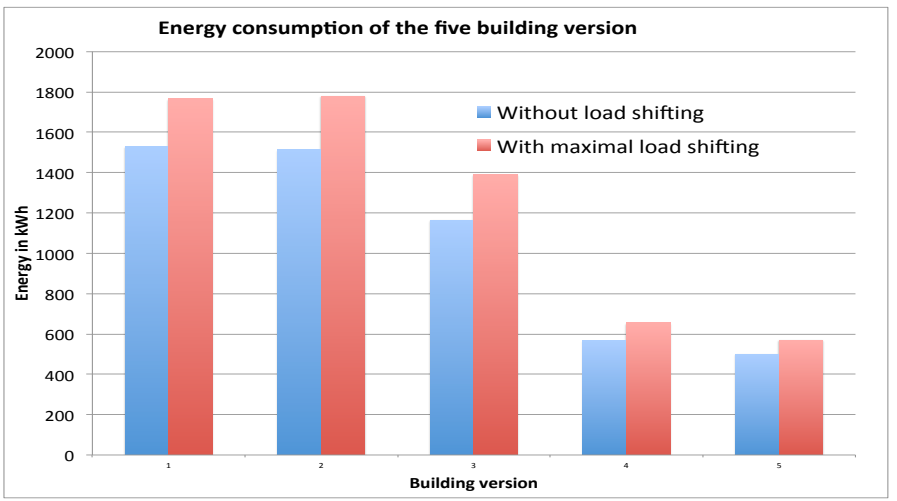

Fig. 3. Energy consumption over one week for the five versions of the building. For each building the consumed energy is displayed without load shifting and with the maximal bearable one.

First, one can see that in terms of energy consumption the first and second versions of the building are quite similar. The adjunction of triple glazed windows induces a significant decreasing of energy consumption $(\approx 30 \%)$. Then, the insulation
TABLE III

VALUE OF THE MAXIMUM LOAD SHIFTING DURATION FOR EACH VERSION OF THE BUILDING.

\begin{tabular}{l|c|c|c|c|c|} 
& $1^{\text {st }}$ & $2^{\text {nd }}$ & $3^{\text {rd }}$ & $4^{\text {th }}$ & $5^{\text {th }}$ \\
\hline Load shifting duration & $15 \mathrm{~min}$. & $20 \mathrm{~min}$. & $20 \mathrm{~min}$. & $4 \mathrm{~h}$. & $6 \mathrm{~h}$.
\end{tabular}

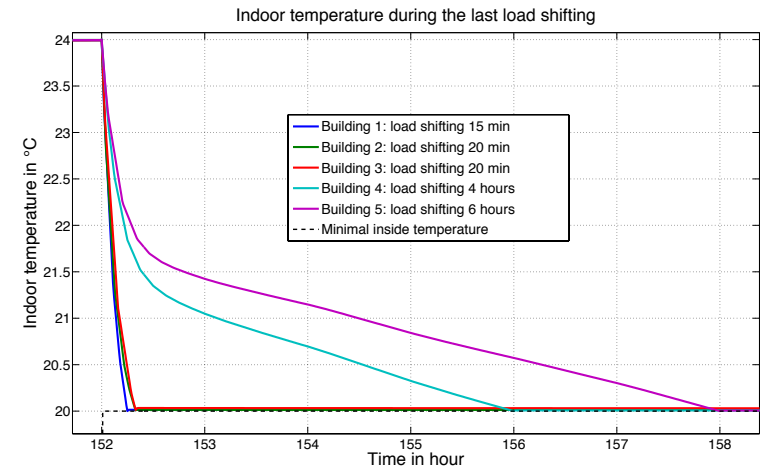

Fig. 5. Comparison of the optimal indoor temperature during the load shifting of the last day of the week

of the external walls allows to clearly decrease the energy consumption $(\approx 50 \%)$. So, the most effective renovation in terms of energy consumption seems to be the increasing of insulation. But, the triple glazed windows also allows to achieve a noticeable decreasing of energy consumption.

Now, considering the ability in handling load shiftings one can see that the three first versions of the building cannot handle load shifting superior to 20 minutes. Interestingly, the adjunction of triple glazed windows does not improve the load shifting ability whereas it is performant for energy saving. Actually, the ability in handling load shifting becomes important when the insulation is increased.

\section{B. Explanation of the results}

As seen on table II, the renovation mainly influences the slow time constant of the systems. It is particularly noticeable that once some insulation has been added to the building the slow time constant is almost twice bigger than the one of the non insulated models. Nevertheless, even for the first version of the building, the slow and medium time constants are large compared to the duration of the maximum bearable load shifting (15 min.). Thus, this modification of the slow and medium time constants through insulation does not satisfyingly explain the results.

Actually, a role is played by the zeros of the transfer function between the heating flux on the air node and the indoor temperature. Table IV exhibits that the slow (resp. medium) zero and pole of the first building version are closer from each other than the ones of the fifth version. This increasing distance between poles and zeros results in an increasing controllability of the slow time scale state variables. This increased controllability allows to store energy within 24 hours in the slow state variables. In contrast the non-insulated building slow time scale are not controllable enough to store 


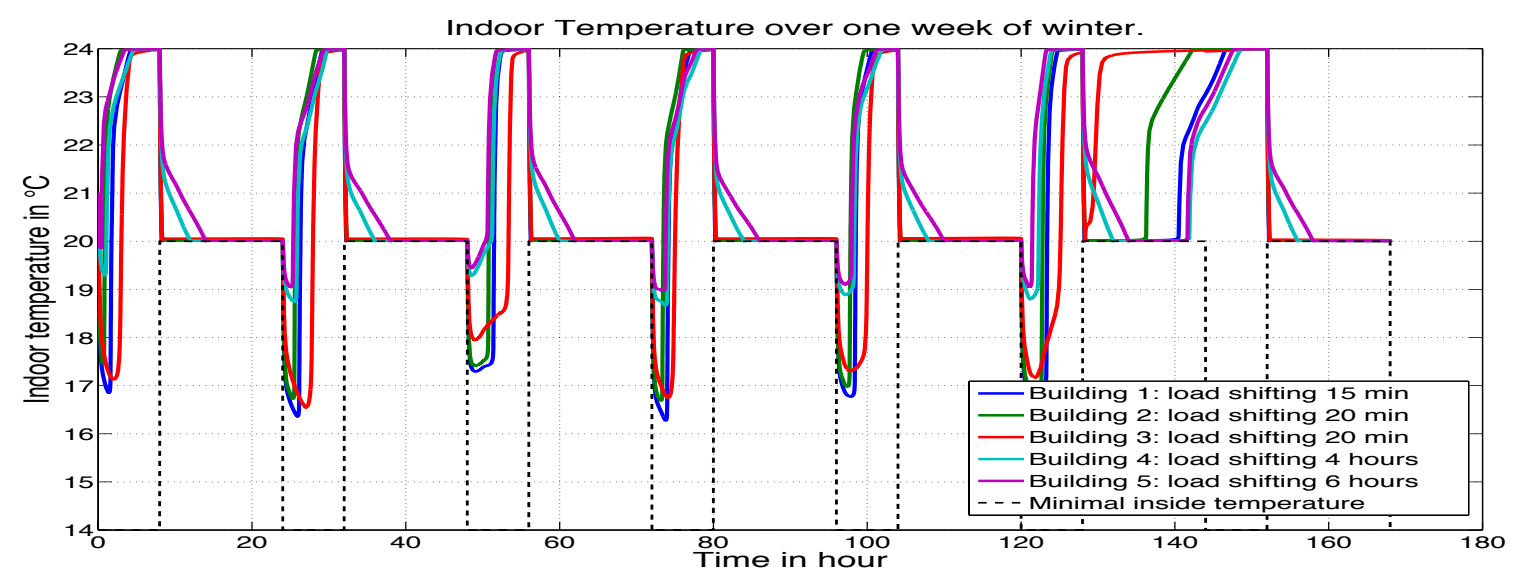

Fig. 4. Comparison of the optimal indoor temperature of each building with the maximum bearable load shiftings duration

energy within 24 hours in the slow time scale state variables, this results in an energy storage in the fast time scale state variable and thus in a small load shifting period.

\section{TABLE IV}

VALUE OF THE POLES AND THE ZEROS OF THE DIFFERENT TIME SCALES FOR THE FIRST AND THE FIFTH VERSION.

\begin{tabular}{c|c|c|} 
& $1^{\text {st }}$ version & $5^{\text {th }}$ version \\
\hline slow pole & $P=-0.0105$ & $P=-0.00558$ \\
slow zero & $Z=-0.0178$ & $Z=-0.0174$ \\
\hline medium pole & $P=-0.0789$ & $P=-0.0548$ \\
medium zero & $Z=-0.268$ & $Z=-0.251$
\end{tabular}

\section{CONCLUSION}

First, The state constraint dynamic optimization is a efficient approach to study intrinsic properties of residential building, to say, in a regulation independent fashion. Plus, the time continuous approach allows to have precise results even when considering fast time scales phenomenom.

Now, considering the load shifting opportunities, we have emphasized that a non insulated residential house cannot handle load shifting superior to 20 minutes even if an advanced strategy of regulation is used. To allow these buildings to handle long load shiftings, their thermal mass is not sufficient. Thus, to achieve long load shifting duration, the buildings must be insulated enough or have auxiliary energy storage capacity. But, the regulation system is not sufficient to achieve long load shifting duration.

\section{REFERENCES}

[1] J. Balcomb, J. Hedstrom, and R. McFarland, "Simulation analysis of passive solar heated buildings-preliminary results," Solar Energy, vol. 19, no. 3, pp. 277-282, 1977.

[2] A. Kojima and M. Morari, "LQ control for constrained continuous-time systems," Automatica, vol. 40, pp. 1143-1155, 2004.

[3] P. Xu, P. Haves, J. Braun, and L. Hope, "Peak demand reduction from pre- cooling with zone temperature reset in an office building," Proceedings of the ACEEE 2004 Summer Study on Energy Efficient in Buildings, 2004.

[4] J. Braun, "Reducing energy costs and peak electrical demand through optimal control of building thermal storage," ASHRAE Transactions, vol. 96, no. 2, pp. 839-84, 1990.
[5] A. Agrachev and Y. Sachkov, Control Theory from the Geometric Viewpoint. Springer, 2004.

[6] J. Bonnans and T. Guilbaud, "Using logarithmic penalties in the shooting algorithm for optimal control problems," Optimal Control Applications and Methods, vol. 24, pp. 257-278, 2003.

[7] L. Lasdon, A. Waren, and R. Rice, "An interior penalty method for inequality constrained optimal control problems," IEEE Transactions on Automatic Control, vol. 12, pp. 388-395, 1967.

[8] P. Malisani, F. Chaplais, and N. Petit, "Design of penalty functions for optimal control of linear dynamical systems under state and input constraints," 50th IEEE Conference on Decision and Control (submitted), 2011.

[9] B. Peuportier and I. Sommereux, "Simulation tool with its expert interface for the thermal design of multizone buildings." International Journal of Sustainable Energy, vol. 8, no. 2, pp. 109-120, 1990.

[10] A. Brun, C. Spitz, and E. Wurtz, "Analyse du comportement de différents codes de calcul dans le cas de bâtiments à haute efficacité énergétique," IXème colloque interuniversitaire Franco-québécois sur la thermique des systèmes, 2009.

[11] H. Khalil, Nonlinear Systems. Prentice Hall, 2002.

[12] P. Malisani, F. Chaplais, N. Petit, and D. Feldmann, "Thermal building model identification using time-scaled identification methods," 49th IEEE Conference on Decision and Control, pp. 308-315, 2010.

[13] K. Zhou, J. Doyle, and K. Glover, Robust and Optimal Control. Prentice Hall, 1996.

[14] A. Forsgren, P. Gill, and M. Wright, "Interior methods for nonlinear optimization," SIAM Review, vol. 4, no. 4, p. 525-597, 2002.

[15] A. Kugi, K. Graichen, N. Petit, and F. Chaplais, "Handling constraints in optimal control with saturation functions and system extension," Systems and Control Letters, vol. 59, no. 11, pp. 671-679, 2010.

[16] A. Bryson and Y. Ho, Applied Optimal Control. Ginn and Company: Waltham, MA, 1969.

[17] L. Shampine, J. Kierzenka, and M. Reichelt, Solving boundary value problems for ordinary differential equations in MATLAB with bvp4c., 2000. [Online]. Available: http://www.mathworks.com/bvp_tutorial 